\title{
APRENDIZAJE A TRAVÉS DE PROYECTOS COLABORATIVOS CON TIC. ANÁLISIS DE DOS EXPERIENCIAS EN EL CONTEXTO EDUCATIVO.
}

\section{LEARNING THROUGH COLLABORATIVE PROJECTS WITH ICT. ANALYSIS OF TWO EXPERIENCES IN THE EDUCATIONAL CONTEXT.}

\author{
Verónica Basilotta Gómez-Pablos; veronicabgp@usal.es \\ Gabriel Herrada Valverde; gabriel.h.valverde@usal.es \\ Universidad de Salamanca
}

\section{RESUMEN}

En el trabajo que se presenta a continuación, se ha asumido la tarea de revisar dos proyectos colaborativos con TIC que se están desarrollando en diversos centros educativos. Se ha realizado un análisis en profundidad en base a unas categorías definidas a priori, en dos proyectos de interés para la Comunidad Educativa, que han recibido premios y que se han implementado en varios cursos académicos: Poesía eres tú y Flautateka. Las conclusiones apuntan en general a una valoración positiva por parte de los miembros del proyecto, que consideran que estas experiencias contribuyen al aprendizaje del alumnado y al desarrollo de sus competencias.

PALABRAS CLAVE: TIC, aprendizaje, educación, trabajo colaborativo, innovación pedagógica.

\section{ABSTRACT}

In the work presented below, it has taken on the task of reviewing two collaborative projects with ICT being developed in various schools. We performed a thorough analysis based on a priori defined categories in two projects of interest to the educational community, which have received awards and have been implemented in various academic courses: Poesía eres tú and Flautateka. The conclusion draw to a positive assesment by project members, who believe that these experiences contribute to student learning and developing their skills.

KEYWORDS: ICT, Learning, Education, Collaborative work, Educational innovation 


\section{INTRODUCCIÓN}

La integración curricular de las TIC en los centros educativos cada día adquiere un mayor protagonismo, por ello, es fundamental que los alumnos estén formados en estas competencias determinadas como básicas, con el objetivo de potenciar en ellos aprendizajes significativos (Canales y Marqués, 2007)

Por otro lado, el uso que se da a estos medios requiere del soporte que ofrece el aprendizaje colaborativo, para optimizar su intervención y formar verdaderos ambientes de aprendizaje que promuevan el desarrollo integral de los estudiantes y sus múltiples capacidades (Calzadilla, 2008)

Como dice Badía (2005: 1) "en la sociedad actual, caracterizada y sustentada principalmente por los procesos de creación, gestión y difusión de la información, cada vez tienen menos cabida las personas que actúan como un ser aislado e independiente. En el polo opuesto se va imponiendo otra perspectiva más social e interdependiente de cada uno de los miembros de una sociedad, organización o grupo; una perspectiva que requiere del individuo la necesidad ineludible de colaboración con los otros"

La escuela por lo tanto, debe responder a estas exigencias de la sociedad actual y enseñar a sus alumnos a colaborar para aprender. Debemos aprovechar las posibilidades que nos ofrecen las nuevas tecnologías para potenciar el aprendizaje colaborativo y constructivista.

\subsection{Aprendizaje colaborativo y TIC}

La enseñanza y aprendizaje en ambientes colaborativos fomenta espacios en los que se desarrollan habilidades individuales y grupales a partir de la discusión entre los alumnos, siendo cada uno responsable de su aprendizaje. Estos ambientes generan múltiples posibilidades y oportunidades de intercambio lo que potencia el crecimiento del grupo (Rubia, Jorrí y Anguita, 2009)

Al abordar el concepto de aprendizaje colaborativo, Johnson y Johnson (1986) destacan las siguientes características que lo definen:

- Interdependencia positiva: los miembros del grupo persiguen un objetivo común, por lo que están motivados en ayudarse mutuamente, en compartir recursos e información.

- Fomento de la interacción: los miembros de un grupo se ayudan unos a otros para trabajar eficientemente, mediante la contribución individual de cada uno.

- Responsabilidad individual: cada uno de los miembros del grupo debe asumir su responsabilidad para contribuir al aprendizaje de todos.

- Desarrollo de competencias: académicas e interpersonales.

- Valoración de los miembros del grupo: mantener una buena relación de colaboración con los demás y estar dispuesto a dar y recibir comentarios y críticas constructivas sobre la contribución individual y del grupo. 
El aprendizaje colaborativo contribuye a la construcción de un conocimiento compartido y las TIC, si se utilizan de manera adecuada, pueden enriquecer estos ambientes. Siguiendo a Calzadilla (2008) las TIC representan ventajas para este aprendizaje porque estimulan la comunicación interpersonal al facilitar el intercambio de información y el diálogo y discusión entre todas las personas implicadas en el proceso; facilitan el trabajo colaborativo, al permitir que los alumnos/as compartan información, trabajen con documentos conjuntos y faciliten la solución de problemas y toma de decisiones; permiten el seguimiento del progreso del grupo, a nivel individual y colectivo; posibilitan el acceso a información y contenidos de aprendizaje; facilitan la gestión y administración de los alumnos; y fomentan la creación de ejercicios de evaluación y autoevaluación, con los que el docente podrá conocer el nivel de logro y rediseñar la experiencia de acuerdo a su ritmo y nivel ofreciendo al alumno/a retroalimentación sobre su desempeño.

\subsection{Proyectos colaborativos con TIC}

Entendemos por Proyecto Colaborativo con TIC en el contexto educativo, un trabajo en el que intervienen un conjunto de individuos con intereses comunes (alumnos, profesores, padres y comunidad educativa) que participan de forma activa a través de actividades colaborativas, entre equipos de personas y dentro de un proceso de aprendizaje utilizando las Nuevas Tecnologías.

Según Vélez (1998) en el aprendizaje a través de proyectos colaborativos se confía en el alumno/a y en la capacidad de exploración de su mundo, lo cual hace que esté motivado y desee desarrollar sus habilidades y destrezas buscando lo mejor de sí.

Badía y García (2006) consideran que en el proceso de enseñanza-aprendizaje basado en la elaboración de proyectos mediante TIC, se produce la siguiente interrelación entre los principales componentes del proceso (alumno, profesor, contenidos):

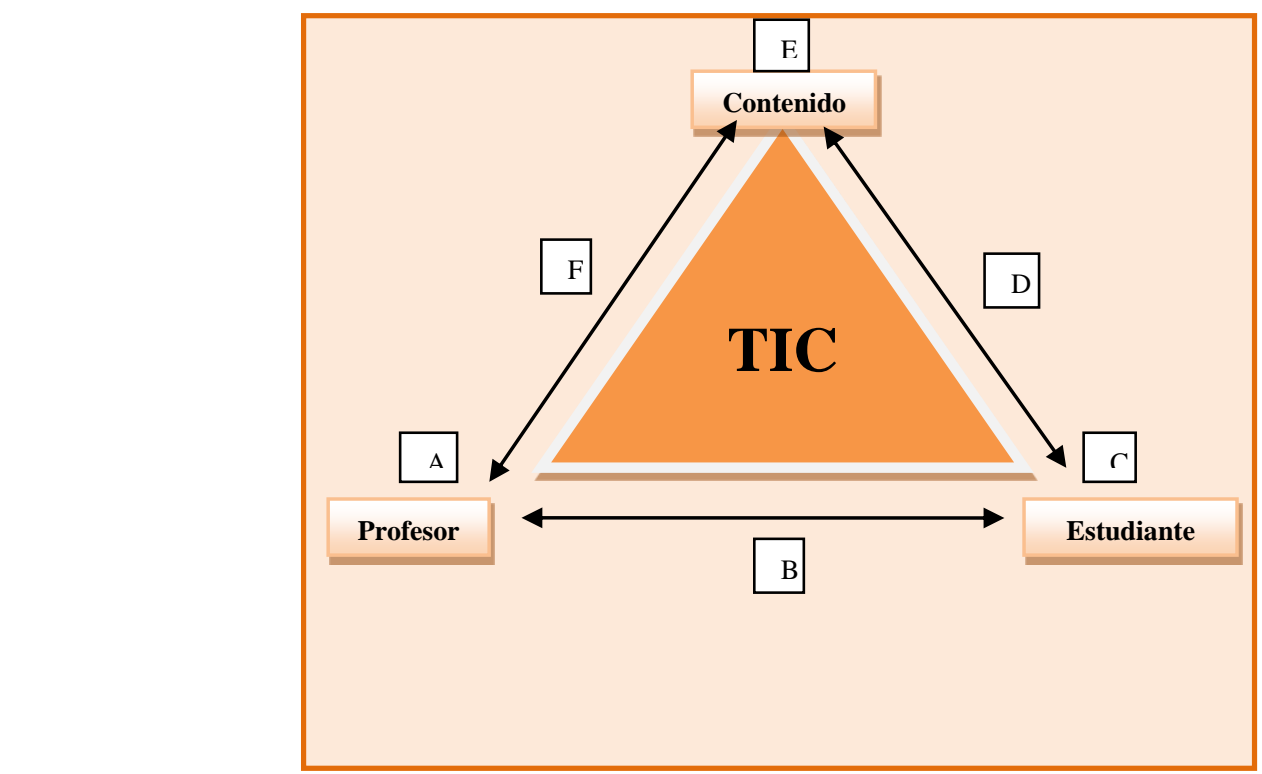

Imagen 1: Interrelación entre los principales componentes del proceso de enseñanza-aprendizaje basad en la elaboración de proyectos mediante las TIC (Badia y Garcia, 2006). 
La tecnología puede ayudar al docente en el diseño o planificación del proyecto, en su desarrollo e implementación y en la evaluación del mismo. Así mismo, puede ser una herramienta que le facilite la interacción educativa con sus estudiantes y con otros docentes. Por otro lado, las TIC pueden contribuir a facilitar el trabajo del estudiante en un doble sentido: por un lado, fomentando su trabajo individual, y por otro, estimulando la interacción educativa con sus compañeros de grupo de trabajo. Finalmente, las TIC pueden ayudar a poner en relación al estudiante con el contenido a través de bases de datos, programas de búsqueda especializada en Internet, programas de clasificación de documentos, procesadores de textos, programas para representar la información, programas para elaborar la información obtenida y programas para comunicar y compartir la información.

Harris (1998) realiza una clasificación de los proyectos educativos telemáticos, nos habla de proyectos centrados en los intercambios interpersonales; en la recopilación de información; y en la resolución colaborativa de problemas.

1. Proyectos centrados en los intercambios interpersonales: el objetivo principal es la comunicación virtual entre los individuos. Estos intercambios pueden ser entre individuo-individuo, individuo-grupo y grupo-grupo.

2. Proyectos centrados en la recopilación y análisis de información. Harris (1995) considera que hay una gran cantidad y variedad de información disponible en Internet, en diferentes formatos, que el alumnado debe seleccionar, analizar y organizar; de esta manera los estudiantes estarán construyendo su conocimiento. El individuo debe transformar la información que encuentra en la red para que se produzca un verdadero conocimiento.

3. Proyectos centrados en la solución colaborativa de problemas. Según Harris (1995) la resolución de problemas es una de las actividades más beneficiosas, que se pueden ofrecer a los estudiantes de cualquier edad. Se trata de proyectos destinados a promover el pensamiento crítico del alumno, la colaboración y el aprendizaje basado en problemas.

En definitiva, la importancia de desarrollar Proyectos Colaborativos con TIC en el contexto educativo y formar al profesorado para ello, radica en que son un recurso metodológico muy adecuado para trabajar y evaluar las competencias del alumno.

\section{OBJETIVOS}

En el estudio que se presenta a continuación se analizan dos proyectos colaborativos con TIC, con el objetivo de examinar su potencial para desarrollar e integrar de forma armónica las competencias del alumnado. La elección de estas experiencias se justifica porque tienen una gran repercusión en la comunidad educativa y difusión en la red, han sido implementados en varios cursos académicos y han obtenido diversos reconocimientos y premios otorgados por instituciones de prestigio. Se pretende: 
a) Presentar dos proyectos colaborativos desarrollados por el profesorado y el alumnado de diversos centros educativos.

b) Mostrar dos experiencias o proyectos relevantes que sirvan de modelo de buenas prácticas con TIC para los docentes.

b) Analizar estas dos experiencias para conocer sus posibilidades educativas, mejorar su desarrollo y evaluación.

\section{METODOLOGÍA}

En lo que se refiere a la naturaleza de la investigación nos hemos decantado por un enfoque principalmente cualitativo, pues consideramos que puede ser más fructífero para entender la relación de estos proyectos con las competencias del alumnado. El análisis se ha llevado a cabo partiendo de los siguientes criterios teórico-prácticos:

\section{Criterios para el análisis de los proyectos}

1. Asunción (o consecución) de las características básicas de la metodología del aprendizaje colaborativo, según Smith (1998) Johnson y Johnson $(2005,2009)$ y Gutiérrez (2011):

1.1. Interdependencia positiva.

1.2. Responsabilidad individual y grupal de los miembros del grupo.

1.3. Liderazgo compartido por todos los integrantes del equipo.

1.4. Papel activo del alumnado en el proceso de construcción de conocimiento.

1.5. Profesorado adopta el rol de guía, orientador y gestor de experiencias de aprendizaje.

2. Uso pedagógico de las herramientas TIC para favorecer el aprendizaje colaborativo (herramientas de la Web 2.0)

2.1. Estimulan la comunicación interpersonal entre todas las personas implicadas en el proceso.

2.2. Facilitan el trabajo colaborativo.

2.3. Permiten el seguimiento del progreso del grupo, a nivel individual y colectivo.

2.4. Posibilitan el acceso a información y contenidos de aprendizaje.

2.5. Permiten evaluar el logro del alumno.

3. Incorporación de la estructura básica del trabajo por proyectos: planificación, desarrollo y evaluación (Tanoni y Alonso, 2005).

\section{RESULTADOS DEL ANÁLISIS}

El proyecto Poesía eres tú ha sido impulsado por los profesores de lengua española y literatura Silvia González Goñi @SilviaGongo y por José Hernández @pephernández. Consiste en elaborar de forma colaborativa un videopoemario con poemas seleccionados, recitados y comentados 
por alumnos de diferentes etapas educativas. De esta manera, consigue acercar la poesía a las aulas de una manera dinámica, activa y participativa. Utiliza un blog como lugar de encuentro y para la puesta en común de las diferentes actividades (ver imagen 2).

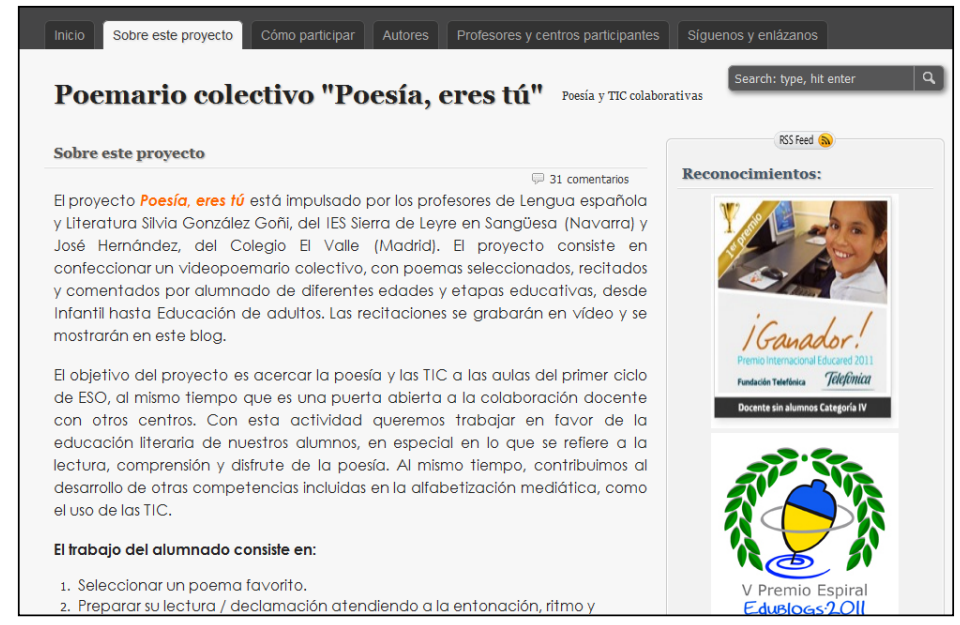

Imagen 2: Blog del proyecto colaborativo Poesía eres tú

En el desarrollo de este trabajo se pone en juego un elemento clave del aprendizaje colaborativo que es la interdependencia positiva. Los alumnos trabajan con el profesorado en la construcción de un poema, que luego grabarán conjuntamente y mostrarán en un blog destinado al proyecto. Todos ellos tienen un objetivo común, una razón de peso para trabajar juntos maximizando su aprendizaje y el de los demás.

En primer lugar, los alumnos realizan una lectura y declamación del texto, posteriormente proceden a la grabación del vídeo con los demás alumnos. Esta grabación implica una asignación de roles para construir un mismo trabajo entre los alumnos: cámara, presentador, estilismo, etc. Se trata de un proceso que se va desarrollando gradualmente siendo los alumnos responsables de su desempeño individual dentro del grupo. Por lo tanto, cada actividad es una realidad individual que ampara detrás un trabajo colectivo.

El liderazgo lo asume principalmente el profesorado sobre todo, cuando se trabaja con alumnos de Infantil y Primaria, no obstante, algunas decisiones son compartidas, ya que los alumnos deben seleccionar el poema que van a trabajar, al igual que la reflexión que se deriva de este trabajo para la construcción de ideas comunes y colaborativas.

Los alumnos en este proceso de aprendizaje participan de forma activa en el trabajo de la poesía en el aula, a) seleccionado un poema favorito, b) preparando su lectura y declamación, c) seleccionando un fragmento favorito del poema y justificando por qué se ha seleccionado, d) preparando una breve exposición sobre el significado del poema, además de una e) breve biografía del autor para finalmente presentarlo ante las cámaras.

Igualmente el docente adopta un rol de guía del alumno en la consecución de la actividad que este último realiza. El docente debe animar, motivar y alentar al alumno para que participe en 
la consecución de los objetivos del proyecto Poesía eres tú. El profesor debe grabar la recitación del poema, editar el vídeo y subirlo finalmente a un servidor como Vimeo o Youtube.

Como se mencionó anteriormente, en este proyecto se utiliza un blog como lugar de encuentro, de comunicación entre los participantes principalmente el profesorado; sin embargo sería fundamental para estimular la interacción entre todos los implicados y aprovechando el potencial educativo de las redes sociales, crear una cuenta del proyecto en Twitter y en Facebook. Aunque el mismo ya dispone de hashtag en twitter: \#poesiaerestu, sería aconsejable también contar con un perfil en ambas redes para que el profesorado pueda interactuar, comunicarse con los demás compañeros, y debatir acerca del proyecto, compartiendo con los demás inquietudes, observaciones, sugerencias, fomentando de este modo un trabajo más enriquecido.

El blog promueve el trabajo colaborativo entre los centros que participan en la elaboración de un videopoemario colectivo, además permite el seguimiento de todo el grupo, pero no de manera individual, en este caso, cada centro puede plasmar su experiencia en su propio blog de aula. Este edublog ${ }^{1}$ en general es bastante intuitivo y recoge las principales características del proyecto, contenidos y bases para participar.

En definitiva, esta experiencia se viene fraguando desde hace unos años, los creadores del mismo empezaron colaborando con actividades semejantes pero a menor escala. Observaron que la reacción de los alumnos cuando participaban de forma colaborativa con otros centros era sumamente positiva; por lo que decidieron crear finalmente las bases de lo que es ahora Poesía eres tú. De forma paralela y según avanzaba el proyecto y la implicación de sus participantes, surgió la idea a través de las redes sociales principalmente Twitter, de abrir el proyecto a más centros y niveles educativos.

En general, el proyecto se ha desarrollado de forma positiva, incorporándose paulatinamente participantes para lograr el producto final que era la elaboración de un videopoemario colectivo.

Con respecto a la evaluación no se especifica cómo debe realizarse, qué estrategias pueden utilizarse para evaluar el desempeño del alumno y qué resultados han obtenido los centros que han participado. Por ejemplo, el profesorado participante podría evaluar a sus alumnos a través de una rúbrica, un portafolio, o un diario de clase. Incluso podría evaluarlos a través de la observación en el aula o entrevistándolos directamente.

Otro proyecto colaborativo que se viene desarrollando actualmente es Flautateka, en el área de Educación Musical. En él participan más de 24 centros educativos de toda España con el objetivo de desarrollar contenidos para el estudio de la flauta dulce. Los alumnos preparan los temas con su flauta, lo graban, editan el vídeo y por último, realizan el post en el blog (ver imagen 3) añadiendo partituras, imágenes, letras y enlaces.

Según Lara (2006) podríamos entender un Edublog como aquellos weblogs cuyo principal objetivo es apoyar un proceso de enseñanza y aprendizaje en un contexto educativo. 


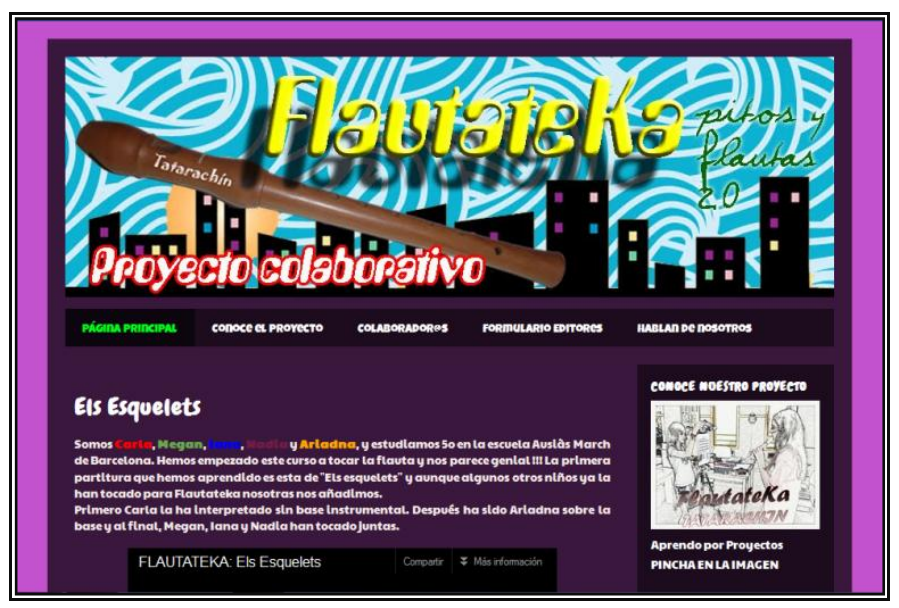

Imagen 3: Blog del proyecto colaborativo Flautateka

Este proyecto fomenta el trabajo colaborativo principalmente entre los individuos del mismo centro educativo. El éxito de todo el grupo depende de la implicación de cada uno de sus miembros, por lo que siguiendo el concepto de interdependencia positiva, los alumnos tienen que adoptar diferentes roles en la misma tarea para conseguir el objetivo final que es la grabación del video. Se deposita cierta responsabilidad individual y colectiva en los discentes, que tienen que desempeñar su rol para alcanzar una meta en conjunto.

El docente debe confiar en las posibilidades del alumno para llevar a cabo la tarea, al mismo tiempo coordina y supervisa todo el proceso, lo guía en su aprendizaje actuando principalmente como líder sobre todo cuando trabaja con alumnos más pequeños.

En relación a las TIC, en el proyecto se utilizan pedagógicamente diferentes herramientas de la Web 2.0 para favorecer la colaboración entre los participantes. Se propone el blog como lugar de encuentro de la experiencia, Google drive y las redes sociales, principalmente Twitter. Sin embargo, en este último, no se establece una verdadera interacción entre los participantes del proyecto, entre otras cosas, porque no todos disponen de cuenta en twitter. Sería fundamental que los docentes y participantes del proyecto pudiesen debatir a través de este medio, sugerir mejoras, interactuar, con el fin de lograr una verdadera comunidad de aprendizaje. El blog del proyecto favorece la comunicación bidireccional, los alumnos junto a sus profesores muestran sus experiencias a los demás participantes pudiendo estos últimos enviar comentarios estableciendo feedback. No obstante, el blog se usa principalmente como espacio guía para la realización de las actividades (a través de indicaciones y tutoriales) y como repositorio de los trabajos realizados por los alumnos de un centro concreto, por lo que se trata en definitiva, de una experiencia colaborativa intracentro, que posteriormente se comparte en el blog.

Este proyecto responde a una planificación previa, consensuada y a unos objetivos definidos a priori que han guiado todo el proceso de enseñanza y aprendizaje:

a) Potenciar el estudio y práctica de la flauta dulce b) crear un banco colaborativo de contenidos multimedia para la flauta dulce c) manejar herramientas colaborativas para 
elaborar materiales educativos d) grabación y edición de vídeos. Así mismo, unas actividades acordes con los objetivos planteados distribuidas en diferentes sesiones: a) sesión Exploratoria, en las que los alumnos buscan una canción para flauta y la ensayan posteriormente; b) sesión Afinamos, los alumnos deberán cumplimentar un formulario, para obtener el permiso de poder publicar su entrada en el blog de Flautateka, la siguiente parte de la sesión se dedicará a conocer las funcionalidades de Blogger, la herramienta que les ayudará a publicar su trabajo en Flautateka; c) sesión Silencio, Grabamos en la que se mostrará a los alumnos como se utiliza la cámara de vídeo/fotos y como realizar la grabación; d) sesión Palomitas y Refrescos se trata de visionar el vídeo para apreciar los aciertos y errores y posteriormente proceder a editarlo a través de Kdenlive (un programa off-line y de software libre) y con las indicaciones del profesor; e) sesión Blogeros se trata de subir finalmente el vídeo editado y revisado por el profesor al blog de Flautateka.

El proyecto en su conjunto se ha ido desarrollando de manera satisfactoria para todos sus participantes, conformándose el blog integrado por diferentes vídeos grabados y editados por los alumnos de los centros educativos e institutos, obteniendo la mención de Buena Práctica con TIC por el Intef (Instituto Nacional de Tecnologías Educativas y de Formación del Profesorado) además del tercer premio en la sexta edición del premio Espiral Edublogs 2012.

En relación a la evaluación sucede lo mismo que en el proyecto anterior, no se menciona en la metodología de trabajo cómo debe evaluarse este proceso de aprendizaje, qué contenidos, competencias, qué herramientas utilizar, etc. Por otro lado, sería recomendable darle un enfoque más interactivo al blog. Aunque los alumnos son los protagonistas de este proceso, participando de manera activa en el blog y convirtiéndose en editores del producto final; en la mayoría de los casos, la plataforma se limita a ser un almacén de contenidos significativos para el estudio de la flauta dulce.

No obstante y a nivel general, la valoración del proyecto es sumamente positiva, con un carácter bastante innovador, motivador para sus alumnos y colaborativo a nivel de centro.

\section{CONCLUSIONES}

Cada vez son más los docentes que planifican y desarrollan proyectos colaborativos con TIC en sus aulas. Hay algunos que se plantean trabajarlos desde diversas disciplinas, otros sin embargo, prefieren abordar una temática más concreta e incluso transversal.

Los dos proyectos descritos anteriormente son un buen ejemplo de experiencias innovadoras en el contexto educativo que tratan de favorecer el trabajo colaborativo entre alumnos y profesores en un contexto de enseñanza y aprendizaje.

"Poesía eres tú", trata de fomentar principalmente la Competencia Lingüística, utilizando la poesía como un medio eficaz para la comunicación oral y escrita, los alumnos expresan e interpretan diferentes versos y transmiten sus emociones a los demás compañeros. 
A través de "Flautateka" sin embargo, se trabaja la Competencia Cultural y Artística. El alumnado disfruta tocando un instrumento como es la flauta dulce, desarrollando sus destrezas más musicales. A través de ese medio, el alumnado puede transmitir pensamientos y sentimientos propios.

A pesar de que cada proyecto se focaliza en un área concreta del currículum, ambas desarrollan otras competencias claves para el alumnado, que enunciamos a continuación:

- Tratamiento de la Información y Competencia Digital

En los dos proyectos el alumnado trabaja con diferentes medios o soportes. Principalmente utilizan recursos tecnológicos para informarse, es decir, acceder a la información, seleccionarla y posteriormente transformarla y comunicarla.

- Competencia para aprender a aprender

Estas experiencias y en general, la metodología a través de proyectos promueven el aprendizaje a lo largo de la vida. Los alumnos trabajan temas relacionados con el mundo real, inician su aprendizaje apoyados por el profesorado que les guía en su aprendizaje, y poco a poco se les va enseñando a ser más autónomos, a ser capaces de dirigir, controlar y gestionar sus conocimientos.

- Autonomía e iniciativa personal

El trabajo por proyectos promueve que el alumno tome decisiones con criterio y las desarrolle asumiendo las consecuencias. Asimismo, el alumno adquiere diversas habilidades como la iniciativa en el trabajo, control emocional y desarrollo de la creatividad.

- Competencia social y ciudadana

Un factor clave en estos proyectos es que fomentan y promueven el trabajo colaborativo entre alumnos y profesores a través del diálogo, el respecto y la participación.

En definitiva, los proyectos colaborativos son una oportunidad para que los alumnos desarrollen sus capacidades y competencias, ofreciéndoles una formación que les sirva para toda la vida.

Podemos concluir que estas experiencias ofrecen las siguientes posibilidades educativas:

- Desarrollan las competencias del alumnado.

- Están centrados en el alumno/a.

- Fomentan el trabajo colaborativo entre alumnos y docentes.

- Fomenta el pensamiento crítico del alumno/a.

- Potencian la autonomía, la responsabilidad individual y la iniciativa en el trabajo.

- Fortalecen la comunicación entre profesores y alumnos a través de múltiples medios.

- Fomentan la interacción y la construcción conjunta de conocimiento.

- Promueven la solución de problemas y la investigación. 
- Fomentan el uso efectivo y eficiente de recursos TIC.

\section{Recomendaciones}

Según hemos revisado diversas experiencias colaborativas en la red, nos hemos encontrado con una serie de cuestiones que es importante que analicemos. En primer lugar, observamos que existen variedad de experiencias que se presentan como proyectos de colaboración con TIC, pero descubrimos que no comparten las mismas características o que el concepto utilizado es diferente. Este aspecto influye en los resultados esperados que no son acordes con el objetivo que se plantea al inicio del proyecto que es la colaboración entre los alumnos, docentes, padres y en definitiva, entre la comunidad educativa a través de las TIC.

En segundo lugar, no observamos una evaluación de los resultados alcanzados, y este es un elemento imprescindible en el proceso de enseñanza y aprendizaje. Se deberían evaluar los aprendizajes de los alumnos respecto a los contenidos abordados teniendo en cuenta no solo los cognitivos, sino los procedimentales y actitudinales (De Benito y Pérez, 2003) La evaluación ha de considerar tanto los aprendizajes en forma de resultados como el proceso. Como decía Piaget "La naturaleza de una realidad viviente no es revelada ni por sus fases iniciales exclusivamente, ni tampoco por las finales, sino por el proceso mismo de sus transformaciones"

Es fundamental incidir en la evaluación de estas experiencias colaborativas con TIC porque le proporcionan al docente una información acerca de los conocimientos adquiridos por los alumnos y la eficacia del proceso de enseñanza y aprendizaje. Asimismo, sería recomendable que el alumno realizase ejercicios de autoevaluación para recibir información sobre su progreso. Todo ello puede ayudar a otros profesores a realizar una actividad similar y a aprender de los éxitos logrados.

Una última cuestión que se ha analizado es la ausencia de estudios cualitativos y cuantitativos que evalúen el impacto de estos proyectos en el aprendizaje y las competencias de los alumnos, reduciéndose la mayoría de los resultados a datos principalmente descriptivos, relacionados con las características de los proyectos. Existen en la red varias plataformas que incluyen estos datos como Eduteka, Colombia Aprende, Educared o Educar.

Como dice Railsback (2002) la planificación y ejecución de un proyecto puede ser un reto al principio pero si al profesorado se le da tiempo y se le aporta ayuda, puede lograr que la educación cobre más vida para sus estudiantes y les motive a tener más iniciativa en su propio aprendizaje.

Lo verdaderamente positivo de estos proyectos colaborativos es que ofrecen la posibilidad de integrar a multitud de docentes, alumnos, padres, profesionales de diferentes disciplinas, lugares, instituciones, creando vínculos duraderos y las bases de una colaboración futura. 


\section{REFERENCIAS BIBLIOGRÁFICAS}

BADÍA, A. (2005). Aprender a colaborar con Internet en el aula. En C. Monereo (Coord.). Internet y competencias básicas. Aprender a colaborar, comunicarse, a participar, a aprender. Barcelona. Editorial: Graó.

BADIA, A., y GARCÍA, C. (2006). Incorporación de las TIC en la enseñanza y el aprendizaje basados en la elaboración colaborativa de proyectos. [Versión electrónica] Revista de Universidad y Sociedad del Conocimiento, RUSC, 3(2), 42-54.

CANALES, R. y MARQUÉS, P. (2007) Factores de buenas prácticas educativas con apoyo de las TIC. Análisis de su presencia en tres centros educativos. Revista Educar, 39, 115-133.

DE BENITO, B. y PÉREZ, A. (2003) La evaluación de los aprendizajes en entornos de aprendizaje cooperativo. En F. Martínez (Comp.). Redes de comunicación en la enseñanza. Las nuevas perspectivas del trabajo corporativo (pp.210-226) Buenos Aires: Paidós.

EDUTEKA (2007). Proyectos colaborativos y cooperativos en Internet. Obtenido 16 Septiembre 2012, desde http://www.eduteka.org/ProyectosColaborativos.php.

HARRIS, J. (1995a): Educational Telecomputing Projects: Interpersonal Exchanges. The Computing Teacher, 6 (22) Obtenido 24 Octubre 2012; desde http://www.ed.uiuc.edu/Mining/March95-TCT.html

HARRIS, J. (1995b): Educational Telecomputing Projects: Information Collections. The Computing Teacher, 7 (22) Obtenido 24 Octubre 2012, desde http://www.ed.uiuc.edu/Mining/April95-TCT.html

HARRIS, J. (1995c): Educational Telecomputing Projects: Problem-Solving Projects. The Computing Teacher, 8 (22) Obtenido 24 Octubre 2012, desde http://www.ed.uiuc.edu/Mining/May95-TCT.html.

JOHNSON, D.W., JOHNSON, R.T. y SMITH, K.A. (1998). Active learning: cooperation in the college classroom. Edina, MN: Interaction Book Company.

JOHNSON, R. T., \& JOHNSON, D. W. (1986). Action research: Cooperative learning in the science classroom. Science and Children (24), 31-32.

LÓPEZ, A. (2006). Construir con otros: proyectos colaborativos para todos los gustos. Obtenido 12 Septiembre 2012, desde Educar, portal educativo del Estado Argentino. Web site: http://portal.educ.ar/debates/educacionytic/inclusion-digital/construir-con-otrosproyectos-colaborativos-para-todos-los-gustos.php

MAENZA, R. R; SGRECCIA, N. F. (2011) Aprendizaje colaborativo mediatizado como estrategia para el desarrollo de competencias: una experiencia con residentes del profesorado de matemáticas. Teoría de la Educación: Educación y Cultura en la Sociedad de la Información, 12 (4), 112-132. 
ROGGI, M. (2008) Internet: un espacio para proyectos colaborativos. Obtenido 12 Septiembre 2012, desde Educar, portal educativo del Estado Argentino. Web site: http://portal.educ.ar/debates/educacionytic/inclusion-digital/internet-un-espacio-para proye.php

RAILSBACK, J. (2002). Project-based instruction: Creating Excitement for Learning. Communities. Portland, OR: Northwest Regional Educational Laboratory. Extraído de http://educationnorthwest.org/webfm_send/460.

RUBIA, B.; JORRÍ, I. y ANGUITA, R. (2009). Aprendizaje colaborativo y TIC. En J. De Pablo Pons (coord.). Tecnología Educativa. La formación del profesorado en la era de Internet. Málaga: Aljibe.

TISCAR, L. (2006) Uso educativo de los blogs. Obtenido 10 Octubre 2012, desde Blogs docentes, Blogs de aula. Web site: http://www.tiscar.com/wpcontent/uploads/2006/12/edublogs tiscar lara dic06.pdf

VÉLEZ, A. (1998). Aprendizaje basado en proyectos colaborativos en la Educación Superior. Obtenido 12 Octubre 2012, desde Universidad EAFIT-UPB-COLCIENCIAS. Proyecto conexiones. Web site: http://www.c5.cl/ieinvestiga/actas/ribie98/190M.html

\section{Para citar este artículo:}

BASILOTTA, V. \& HERRADA, G. (2013). Aprendizaje a través de proyectos colaborativos con TIC. Análisis de dos experiencias en el contexto educativo. EDUTEC, Revista Electrónica de Tecnología Educativa, 44. Recuperado el dd/mm/aa de http://edutec.rediris.es/Revelec2/Revelec44/aprendizaje proyectos colaborativos TIC experiencias.ht $\underline{\mathrm{ml}}$ 